the graph and its asymptote would clearly give the value of $\nu$. If, on the other hand, the graph in question shows no asymptote, that fact signifies that $\mu=1$. In that case the left member of (13) is to be plotted. Since $\nu$ is now not equal to unity this graph will show an asymptote, the slope and intercept of which determine $h$ and $\nu$.

HARVARD UNIVERSITY

\title{
A SERIES OF INVOLUTORIAL CREMONA SPACE TRANSFORMATIONS DEFINED BY A PENCIL OF RULED CUBIC SURFACES*
}

\section{BY AMOS BLACK}

1. Introduction. A series of involutorial Cremona transformations of space were defined by Snydert by means of a correspondence between the surfaces of a pencil of ruled surfaces and the points of a rational curve, called the director curve. The director curve was a part of the basis of the pencil and two of the chief characteristics of the transformation were: one of the principal surfaces was a ruled surface $R$, all of whose generators were parasitic lines; all the tangent planes of the surfaces of the homaloidal web along a certain curve were fixed, being determined by the surface $R$.

In this paper we shall define a series of transformations by means of a correspondence between the surfaces of a pencil of ruled cubic surfaces and the points of certain rational curves. The director curve is not part of the basis of the pencil; one of the principal surfaces is a ruled surface $R$, all of whose generators are parasitic lines; all the surfaces of the homaloidal web have fixed tangent planes along a certain curve, but none of the fixed planes are determined by $R$.

2. Definition of the Transformation. Given a pencil of ruled cubic surfaces $\left|F_{3}\right|$ whose basis curve consists of a double line $d$

* Presented to the Society, September 13, 1935.

$\dagger$ Virgil Snyder, On a series of involutorial Cremona transformations of space defined by a pencil of ruled surfaces, Transactions of this Society, vol. 35 (1933), pp. 341-347. 
and a rational space quintic curve $\delta_{5}$ which has $d$ for a quadrisecant; also, given a rational space curve $r_{m}$, of order $m$, which meets $d$ and $\delta_{5}$ in $m-1$ and $m+1$ points, respectively; then a general surface $F_{3}$ of the pencil meets $r_{m}$ in $3 m-1$ fixed points and in just one general point $O$. Thus there is a $(1,1)$ correspondence between the surfaces of $\left|F_{3}\right|$ and the points $O$ of $r_{m}$. We define $F_{3}: O$ and $O$ as associated surface and point.

A point $P(y)$ in space will determine a unique surface $F_{3}$ of the pencil, hence a unique point $O(z)$ on $r_{m}$. The line $P O$ meets $F_{3}$ in $P, O$, and one other point $P^{\prime}(x)$, which is defined as the image of $P$. Then on each line of the complex of lines meeting $r_{m}$ lies a pair of points $P, P^{\prime}$ in involution.

We shall discuss the case where $m=2$ in detail, and then indicate results for $m$ general.

3. Equations of the Transformation. Let the equations of $d$ and $r_{2}$ be

$$
\begin{array}{ll}
d: & x_{3}=0, x_{4}=0, \\
r_{2}: & x_{1} x_{4}-x_{3}{ }^{2}=0, x_{2}=0 .
\end{array}
$$

Then $D=\left[r_{2}, d\right]=(1,0,0,0)$, and any point on $r_{2}$ can be represented by

$$
O(z)=O\left(z_{1}, z_{2}, z_{3}, z_{4}\right)=\left(\lambda^{2}, 0, \lambda \mu, \mu^{2}\right) .
$$

Let the pencil of surfaces $\left|F_{3}\right|: d^{2} \delta_{5}$ be

$$
\mu F(x)-\lambda F^{\prime}(x)=0,
$$

where $\lambda, \mu$ in (4) is the same as in (3). If $F(O): O$, then

$$
\begin{aligned}
F(x)= & {\left[a_{3}^{\prime} x_{1}+a_{2} x_{2}+b_{1}^{\prime} x_{3}+\left(b_{3}^{\prime}+a_{4}^{\prime}-b_{1}\right) x_{4}\right] x_{3}{ }^{2} } \\
& +\left[b_{1} x_{1}+b_{2} x_{2}+\left(b_{4}^{\prime}+c_{1}^{\prime}\right) x_{3}+b_{4} x_{4}\right] x_{3} x_{4} \\
& +\left[c_{1} x_{1}+c_{2} x_{2}+c_{4}^{\prime} x_{3}\right] x_{4}{ }^{2} \\
F^{\prime}(x)= & {\left[a_{2}^{\prime} x_{2}+a_{3}{ }^{\prime} x_{3}+a_{4}{ }^{\prime} x_{4}\right] x_{3}{ }^{2} } \\
& +\left[b_{1}{ }^{\prime} x_{1}+b_{2}^{\prime} x_{2}+b_{3}^{\prime} x_{3}+b_{4}^{\prime} x_{4}\right] x_{3} x_{4} \\
& +\left[c_{1}^{\prime} x_{1}+c_{2}^{\prime} x_{2}+\left(c_{1}+b_{4}\right) x_{3}+c_{4}^{\prime} x_{4}\right] x_{4}{ }^{2} .
\end{aligned}
$$

Denote by $A, B, C$ and $A^{\prime}, B^{\prime}, C^{\prime}$ the coefficients (containing $x_{1}$ and $x_{2}$ only) of $x_{3}^{2}, x_{3} x_{4}, x_{4}^{2}$ in $F(x)$ and $F^{\prime}(x)$, respectively, (that is, $A=a_{3}^{\prime} x_{1}+a_{2} x_{2}$ ); and by $\alpha, \beta, \gamma$ the determinants 


$$
\left|B C^{\prime}\right|=B C^{\prime}-B^{\prime} C,\left|A C^{\prime}\right|,\left|A B^{\prime}\right|
$$

and let

$$
\bar{\mu}=A^{\prime} x_{3}^{2}+B^{\prime} x_{3} x_{4}+C^{\prime} x_{4}^{2} .
$$

Any point on the line joining $P(y)$ to $O(z)$ has coordinates

$$
x_{i}=\rho y_{i}+\sigma z_{i}, \quad(i=1,2,3,4) .
$$

The value of $\rho / \sigma$ for $P^{\prime}(x)$ is given by

$$
\mu F(\rho y+\sigma z)-\lambda F^{\prime}(\rho y+\sigma z)=0 .
$$

Since $P$ is on (4) and $O$ is on $r_{m}$, we find

$$
\rho\left[\mu F(z, y)-\lambda F^{\prime}(z, y)\right]+\sigma\left[\mu F(y, z)-\lambda F^{\prime}(y, z)\right]=0,
$$

where $F(y, z), F^{\prime}(y, z)$ are the first polars of $F(y), F^{\prime}(y)$ with respect to $(z)$, and $F(z, y), F^{\prime}(z, y)$ are the first polars of $F(z), F^{\prime}(z)$ with respect to $(y)$, and $\lambda / \mu=F(y) / F^{\prime}(y)$. Hence

where

$$
\frac{\rho}{\sigma}=-\frac{L_{16}}{K_{11}},
$$

$$
\begin{aligned}
& L_{16}=F^{\prime}(y) F(y, z)-F(y) F^{\prime}(y, z), \\
& K_{11}=F^{\prime}(y) F(z, y)-F(y) F^{\prime}(z, y) .
\end{aligned}
$$

However $L_{16}$ and $K_{11}$ are factorable, and

$$
K_{11}=R_{4} K_{7}, \quad L_{16}=R_{4} F^{\prime} L_{9},
$$

where $F^{\prime}=F^{\prime}(y)$ and

$$
\begin{aligned}
R_{4}= & y_{4} F-y_{3} F^{\prime}, K_{7}=\bar{\mu}\left(\gamma y_{3}^{2}+2 \beta y_{3} y_{4}+\alpha y_{4}^{2}\right)+F_{7}(y), \\
L_{9}= & \bar{\mu} \bar{L}_{6}+F_{9}(y), \\
\bar{L}_{6}= & \left(A \gamma+A^{\prime} \beta\right) y_{3}^{3}+\left(A \beta+A^{\prime} \alpha+B \gamma+B^{\prime} \beta\right) y_{3}^{2} y_{4} \\
& +\left(B \beta+B^{\prime} \alpha+C \gamma+C^{\prime} \beta\right) y_{3} y_{4}^{2}+\left(C \beta+C^{\prime} \alpha\right) y_{4}^{3},
\end{aligned}
$$

and where $F_{7}(y), F_{9}(y)$ are terms containing $y_{3}, y_{4}$ to higher degree than 4 and 5 , respectively. On removing the factor $R_{4}$, the involutorial transformation is therefore expressed by

$$
I_{13}: \quad x_{i}=y_{i} F^{\prime} L_{9}-K_{7} z_{i}, \quad(i=1,2,3,4) .
$$

It is evident from equations (12) that $K_{7}=0$ is the surface 
of invariant points, that $L_{9}=0$ is the image of $r_{2}$, and that $F^{\prime}=0$ is the image of $D$.

4. Images of the Fundamental Elements. Through a general point $O$ of $r_{2}$ passes a generator $g$ of $F(O)$. If $P$ is any point on $g$, since $P O$ lies on $F(O)$, then $P^{\prime}$ is indeterminate, and the line $g$ is parasitic. As $O$ traces $r_{2}$, the line $g$ generates the surface $R_{4}=0$. Since all the generators of $R_{4}$ are parasitic lines, the surface should appear as a factor of the transformation, as we have seen from (10).

Consider a general point $P$ on $d$. It remains invariant on every $F(O)$ of the pencil, except when $P O$ is a generator $g_{o}$ on $F(O)$, hence a generator of $R_{4}$. Thus the total image of $d$ is $R_{4}$.

Since $D$ is a double point on $F(D) \equiv F^{\prime}$, every point of $F^{\prime}$ is transformed into $D$, hence $D \sim F^{\prime}$.

The tangent plane of $F(O)$ at $O$ intersects $F(O)$ in a generator $g_{O}$ and a conic $c_{2}: O$. Every point of $c_{2}$ is transformed into $O$, hence $O \sim c_{2}$. As $O$ traces $r_{2}$, the $c_{2}$ generates the surface $L_{9}=0$. The point $O$ is invariant in the direction of the tangent of $c_{2}$, hence $L_{9}$ and $K_{7}$ are tangent along $r_{2}$.

On every generator of the rational cone with vertex $P$ on $\delta_{5}$ and standing on $r_{2}$ is one point $P^{\prime}$, the image of $P$. The locus of $P^{\prime}$ is a curve $c$ of order two increased by the number of times $P O$ is tangent to $F(O)$ at $P$. Given an arbitrary point $O$ on $r_{2}$, the tangent plane of $F(O)$ at $P$ cuts $r_{2}$ in two points $M$. Given a point $M$, there is a unique surface for which $M P$ is a tangent at $P$, thus one point $O$. This $(1,2)$ correspondence on $r_{2}$ has three coincidences, hence $P \sim c_{5}: P^{3}$. However, through $P$ passes one generator $g_{O}$ of $F(O)$. Hence $c_{5}$ is composite and consists of $g_{0}$ and a $c_{4}: P^{2}$. As $P$ traces $\delta_{5}$ the $g_{0}$ generates $R_{4}$, and $c_{4}$ generates a surface $\Delta_{22}=0$. Since $R_{4}$ factors out of the transformation, the total image of $\delta_{5}$ is $\Delta_{22}$. The equations of $\Delta_{22}$ are found by finding the image of $F(x)$. We see that

$$
F(x): d^{2} D^{2} \delta_{5} \sim F R_{4}{ }^{2} F^{\prime 2} \Delta_{22},
$$

where

$$
\Delta_{22}=\bar{\mu}^{4} \bar{L}_{6}\left(\beta^{2}-\alpha \gamma\right)+F_{22}(y),
$$

and $F_{22}(y)$ contains $y_{3}, y_{4}$ to degree higher than eleven. The point $P$ is invariant in the directions of the two tangents of $c_{4}: P^{2}$, 
hence two sheets of $\Delta_{22}$ are tangent to the two sheets of $K_{7}$ along $\delta_{5}$.

5. Contact Along $d$. The two tangent planes of $F^{\prime}=0$ at any point on $d$ are identical with those of $\mu=0$. It is evident from equations (11), (12), and (13) that each tangent plane of $\bar{\mu}$ (hence of $F^{\prime}$ ) is the tangent plane of one sheet of $K_{7}=0$, one sheet of $L_{9}=0$, two sheets of a general homaloid $S_{13}=0$, and four sheets of $\Delta_{22}=0$ at every point on $d$. Also the remaining three sheets of $L_{9}, S_{13}$, and $\Delta_{22}$ have fixed tangent planes along $d$, the three planes being identical with the tangent planes of $\vec{L}_{6}$ along $d$. The image due to the former contact is $F^{\prime}$ and the latter is $R_{4}$.

Although every tangent plane of $L_{9}, S_{13}$, and $\Delta_{22}$ is fixed at any point on $d$, they are all distinct from the tangent planes of $R_{4}$ at the point. The tangent planes of $F^{\prime}$ at $D$ are

$$
y_{4}=0, \quad \pi=b_{1}^{\prime} y_{3}+c_{1}^{\prime} y_{4}=0 .
$$

At $D$, one of the three tangent planes of $L_{9}, S_{13}, \Delta_{22}$ coincides with $\pi$.

6. Determination of the Parasitic Lines. We have seen that every generator of $R_{4}$ is a parasitic line. In general none of these generators lies on any other surface of the transformation. We wish to find which of these lines do lie on other surfaces, and any other parasitic lines which may arise.

Given a point $O$ on $r_{2}$, the generator $g_{O}$ on $F(O)$ intersects $d$ in a point $L$. The surface $F(O)$ has two pinch points $M$ on $d$. If a point $L$ and a point $M$ coincide, then a generator $g_{o}$ on $F(O)$ passes through a pinch point of $F(O)$. In this case $g_{O}$ is not only parasitic but is a line of contact for $S_{13}, L_{9}, K_{7}, R_{4}, \Delta_{22}$.

Given a point $L$ there are two points $M$. Given a point $M$, there are two surfaces for which $M$ is a pinch point, hence two points $L$. This $(2,2)$ symmetric correspondence on $d$ has two coincidences, hence there are two such parasitic lines. Denote them by $2 g$.

The surface $F^{\prime}$ has two generators passing through $D$, its associated point. Each is a parasitic line. One lies in the plane $y_{4}=0$, hence lies on $R_{4}$, and factors out of the transformation. The other lies in the plane $\pi$ and is a parasitic line for the transformation. This line $g^{\prime}$ is simple on $S_{13}, K_{7}, F^{\prime}$, and $\Delta_{22}$. 
Since $\delta_{5}$ is rational and has $d$ for quadrisecant, the surface of trisecants is a ruled surface of order eight, $R_{8}: \delta_{5}^{3} d^{4}$. Since $r_{2}$ meets $d$ once and $\delta_{5}$ three times there are three trisecants of $\delta_{5}$ which meet $r_{2}$. If $O_{1}$ is a point on $r_{2}$ through which passes a trisecant of $\delta_{5}$, then the trisecant lies on $F\left(O_{1}\right)$ and is parasitic. Thus there are three such parasitic lines, $3 g^{\prime \prime}$. They are simple on $S_{13}, L_{9}, K_{7}$ and triple on $\Delta_{22}$.

7. Table of Characteristics. The images of planes and of fundamental elements can now be expressed by the following table:

$$
\begin{aligned}
& S_{1} \sim S_{13}: r_{2}^{2} d^{7+2 t+2 t+3 t^{\prime}} D^{7} \delta_{5}^{4} 2 g g^{\prime} 3 g^{\prime \prime}, \\
& r_{2} \sim L_{9}: r_{2} d^{5+t+t+3 t^{\prime}} D^{5} \delta_{5}^{3} 2 g 3 g^{\prime \prime}, \\
& d \sim R_{4}: r_{2} d^{3} D^{3} \delta_{5} 2 g \text {, } \\
& D \sim F^{\prime}: d^{2+t+t} D^{2} \delta_{5} g^{\prime}, \\
& \delta_{5} \sim \Delta_{22}: r_{2}^{4} d^{11+4 t+4 t+3 t^{\prime}} D^{11} \delta_{5}{ }^{7} 2 g g^{\prime} 3 g^{\prime \prime 3}, \\
& K_{7}: r_{2} d^{4+t+t} D^{4} \delta_{5}^{2} 2 g g^{\prime} 3 g^{\prime \prime}, \\
& J_{48}=L_{9} R_{4}^{2} F^{\prime 3} \Delta_{22},
\end{aligned}
$$

where the $t$ and $t^{\prime}$ in the multiplicity of $d$ stands for the contact as given in $\$ 5$.

Thus the complete intersection of two surfaces of the web may be written

$$
\left[S_{13}, S_{13}^{\prime}\right]=C_{13} r_{2}^{4} d^{49+4+4+3} \delta_{5}{ }^{16} 2(2 g) g^{\prime} 3 g^{\prime \prime} .
$$

8. The Transformation for $m$ General. We replace $r_{2}$ by a rational curve $r_{m}$, which meets $d$ and $\delta_{5}$ in $m-1$ and $m+1$ points, respectively, with parametric representation

$$
z_{i}=z_{i}(\lambda, \mu), \quad(i=1,2,3,4),
$$

where the $z_{i}$ are homogeneous functions of degree $m$ in $\lambda, \mu$. Denote the $m-1$ points of intersection of $r_{m}$ with $d$ by $D_{i}$, and the associated surfaces by $F^{(i)}$. The transformation has the form

$$
\begin{array}{r}
I_{6 m+1}: x_{i}=y_{i} F^{(1)} F^{(2)} \cdots F^{(m-1)} L_{3 m+3}- \\
K_{3 m+1} z_{i}\left(F, F^{\prime}\right), \\
(i=1,2,3,4),
\end{array}
$$

where $K_{3 m+1}=0$ is the surface of invariant points, $L_{3 m+3}=0$ is the image of $r_{m}$, and $F^{(i)}=0$ is the image of $D_{i}$. The image of $d$ is $R_{4}=0$, as before. The image of a general point $P$ on $\delta_{5}$ is a curve 
$c_{2 m}: P^{m}$. As $P$ traces $\delta_{5}$, the $c_{2 m}$ generates a surface $\Delta_{12 m-2}$, the total image of $\delta_{5}$.

Each of the two tangent planes of each of the $m-1$ surfaces $F^{(i)}$ at each point of $d$ is the tangent plane of one sheet of $L_{3 m+3}$, one sheet of $K_{3 m+1}$, two sheets of a general $S_{6 m+1}$, and four sheets of $\Delta_{12 m-2}$. Also the remaining three sheets of $L_{3 m+3}, S_{6 m+1}$, and $\Delta_{12 m-2}$ have fixed tangent planes along $d$.

The parasitic lines are as follows: $2 g$, two generators of $R_{4}$ which are simple lines of contact for $L, K, S, R, \Delta ;(m-1) g^{\prime}$, $m-1$ lines, one on each $F^{(i)}$, and simple on $S, K, \Delta ;(m+1) g^{\prime \prime}$, trisecants of $\delta_{5}$ meeting $r_{m}$, simple on $S, L, K$ and triple on $\Delta$.

The results can be expressed by the following table:

$$
\begin{aligned}
& S_{1} \sim S_{6 m+1}: r_{m}^{2} d^{4 m-1+2 t_{i}+2 t_{i}+3 t^{\prime}} D_{i}^{4 m-1} \delta_{5}{ }^{2 m} 2 g(m-1) g^{\prime}(m+1) g^{\prime \prime}, \\
& r_{m}=L_{3 m+3}: r_{m} d^{2 m+1+t_{i}+t_{i}+3 t^{\prime}} D_{i}{ }^{2 m+1} \delta_{5}{ }^{m+1} 2 g(m+1) g^{\prime \prime} \text {, } \\
& d \sim \quad R_{4}: r_{m} d^{3} D_{i}{ }^{3} \delta_{5} 2 g \\
& D_{j} \sim F^{(j)}: d^{2+t_{j}+t_{j}} D_{j}{ }^{2} \delta_{5} g_{j}^{\prime} \text {, }
\end{aligned}
$$

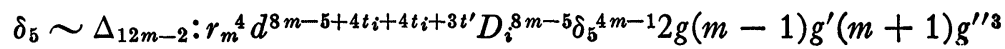

$$
\begin{aligned}
& K_{3 m+1}: r_{m} d^{2 m+t_{i}+t_{i}} D_{i}{ }^{2 m} \delta_{5} 2 g(m-1) g^{\prime}(m+1) g^{\prime \prime} \text {, } \\
& J_{24 m}=L_{3 m+3} R_{4}^{2} F^{(1)^{3}} \ldots F^{(m-1)^{3} \Delta_{12 m-2}} \text {, }
\end{aligned}
$$

where the $t_{i}$ and $t^{\prime}$ represent the contact along $d$, and $i=1,2, \cdots, m-1$.

The complete intersection of two surfaces of the web is

$$
\begin{aligned}
& {\left[S_{6 m+1}, S_{6 m+1}^{\prime}\right]} \\
& \quad=C_{6 m+1} r_{2}^{4} d^{(4 m-1)^{2}+(m-1)(4+4)+3} \delta_{5}(2 m)^{2} 2(2 g)(m-1) g^{\prime}(m+1) g^{\prime \prime} .
\end{aligned}
$$

Cornell University 